\title{
The Asymptotics of the Correlations Functions in $(1+1) d$ Quantum Field Theory From Finite Size Effects in Conformal Theories
}

\author{
A.Mironov \\ Department of Theoretical Physics \\ P.N.Lebedev Physical Institute \\ Leninsky prospect, 53, Moscow, 117 924円 \\ A.Zabrodin \\ Institute of Chemical Physics \\ Kosygina st., 117334, Moscow
}

${ }^{1}$ E-mail address: theordep@sci.fian.msk.su 


\begin{abstract}
Using the finite-size effects the scaling dimensions and correlation functions of the main operators in continuous and lattice models of 1d spinless Bose-gas with pairwise interaction of rather general form are obtained. The long-wave properties of these systems can be described by the Gaussian model with central charge $c=1$. The disorder operators of the extended Gaussian model are found to correspond to some non-local operators in the $X X Z$ Heisenberg antiferromagnet. Just the same approach is applicable to fermionic systems. Scaling dimensions of operators and correlation functions in the systems of interacting Fermi-particles are obtained. We present a universal treatment for $1 d$ systems of different kinds which is independent of the exact integrability and gives universal expressions for critical exponents through the thermodynamic characteristics of the system.
\end{abstract}




\section{Introduction}

Recently the new effective method to investigate $1 d$ quantum systems using the finite-size effects in conformal field theory (CFT) was proposed in a number of papers [1-6]. In $1 d$ quantum systems there exist phase transition at zero temperature and corresponding long-distance conformal symmetry appears to be enough to determine the properties independent of the interaction at small scales. These properties are really of a great interest in the $1 d$ systems. Among them the correlation functions, i.e. expectation values of operator products in different points are especially interesting. The correlations at large distances decrease as a power of the distance (at zero temperature) the "critical exponents" being continuously dependent on the coupling constant [7-9].

In $2 d$ systems the conformal invariance is known to constrain the spectrum of scaling dimensions [10]. It does really determine the critical exponents in $1 d$ quantum systems (i.e. in $2 d$ models of the quantum field theory with one space and one time directions). The essential point is that it is possible to calculate central charge and scaling dimensions of the effective long-distance conformal theory using the so-called finite-size effects $[1,2,11]$.

This paper is a review of applications of CFT methods to "real" physical $1 d$ systems which, as they appear, are neither conformal nor exactly integrable. We propose a unified treatment of physically relevant $1 d$ systems of different kinds. Among them are continuous and lattice models of Bose or Fermi multiparticle systems with interaction of a quite general type and $1 d$ lattice antiferromagnetic spin chains. We shall study the long-wave behaviour of the correlation functions in these models. The corresponding critical exponents are determined from the leading finite-size corrections to the low energy spectrum. A remarkable point is that these leading corrections are in a sense universal: they are completely determined by general thermodynamic characteristics of the systems. So our expressions for the critical exponents through certain susceptibilities have a wide range of applicability. They include all previously known examples as particular cases.

This review is based mostly on our short publications $[22,23,43]$. Here we present the more detailed argumentation with new examples and discussions.

To begin with, let us remind the ideology and main formulas of the finite-size approach in CFT [11]. Consider a CFT on an infinite strip of width $L$ in space direction. Then each 
primary conformal operator $\phi$ gives rise to an infinite "tower" of the eigenstates $\left|\phi_{k, \bar{k}}\right\rangle$ of the Hamiltonian with energies

$$
E_{L}^{\phi}(k, \bar{k})=E_{L}^{v a c}+\frac{2 \pi v}{L}(h+k+\bar{k})
$$

and momenta

$$
P_{L}^{\phi}(k, \bar{k})=P_{\infty}^{\phi}+\frac{2 \pi}{L}(s+k-\bar{k})
$$

(such that $\left\langle\operatorname{vac}|\phi| \phi_{k, \bar{k}}\right\rangle \neq 0$ ). Here $h \equiv \Delta+\bar{\Delta}$ and $s \equiv \Delta-\bar{\Delta}$ are scaling dimension and spin of the operator $\phi$ respectively, $k, \bar{k} \geq 0$ - are integers, $E_{L}^{v a c}$ is the ground state energy of the system in the "box" of length $L$. The momentum of the lowest state $\left|\phi_{0, \overline{0}}\right\rangle \equiv|\phi\rangle$ of the tower at $L \rightarrow \infty$ is denoted by $P_{\infty}^{\phi}$. At last, the parameter $v$ in (1) takes into account the difference in units of the space and temporal quantities, i.e. $v$ is merely the velocity of sound excitations (group velocity at the Fermi surface).

Thus, to find the spectrum of primary operators it is sufficient to calculate the energies of the lowest excitations $E_{L}^{\phi}(0,0) \equiv E_{L}^{\phi}$ of each tower up to the terms of order $L^{-1}$

$$
E_{L}^{\phi}-E_{L}^{v a c}=2 \pi v h L^{-1}
$$

The states from $\phi$-tower with $k, \bar{k} \neq 0$ correspond to the so-called descendants (or secondary fields) of $\phi[10]$. They have the definite scaling dimensions $h+k+\bar{k}$ (but they are not primary fields!) and give a contribution to correlators too. However, we shall see that the leading term of asymptotics of the correlators is determined only by the primary fields.

The long-wave asymptotics of two-point equal time correlator of the field $\phi$ has the form

$$
\langle\phi(x) \phi(0)\rangle \sim \cos \left(P_{\infty}^{\phi} x\right) x^{-2 h}
$$

the oscillating factor $\cos \left(P_{\infty}^{\phi} x\right)$ being determined by a gap $P_{\infty}^{\phi}$ into the momentum spectrum. For simplicity, throughout this paper we consider only the equal time correlators. Their asymptotics is completely determined by the scaling dimensions $h$ of the field $\phi$. 
The generalization to the space-time correlators is straightforward. In this case one also should determine the value of spin $s$.

Indeed, the finite-size approach allows one to find out the central charge of the Virasoro algebra of the conformal symmetry using the equation similar to eqs.(1)-(3). Namely, the correction $\sim L^{-1}$ to the ground state energy of the system is proportional to the central charge $c[1,2]$. For example, for the theory with periodic boundary conditions one obtains:

$$
E_{L}^{v a c}=\epsilon_{0} L-\frac{\pi c v}{6 L} .
$$

Here $\epsilon_{0}$ is the energy of the ground state per unit length in an infinite volume. The first term in (5) depends on regularization, but the second does not. In other words, the first (thermodynamic) term is determined by ultraviolet behaviour of the system; the leading corrections being given by the infrared properties.

Usually it is convenient to calculate the central charge by means of another formula which is equivalent to (5). Due to the modular invariance the case of zero temperature $(T=0)$ and large $L$ is equivalent to the case of the infinite volume $(L=\infty)$ and low temperature $T$. In the latter case (5) is rewritten as follows:

$$
f(T)=\epsilon_{0}-\frac{\pi c T^{2}}{6 v}
$$

where $f(T)$ is the density of the free energy as a function of the temperature (at small $T)$.

In the paper [3-6] the equations (1)-(3),(5)-(6) were used to calculate the critical exponents in $1 d$ exactly solvable models. Namely, the low-lying excitation energies have been calculated within the Bethe-ansatz framework [12]. At present time there exist many papers devoted to this topic (see, for example, [1-6,13-20] and references therein) and we would not repeat here the finite-size calculations in integrable theories. Instead, we shall concentrate on the following important but less familiar aspects. First, the method turns out to be applicable to the models with general interactions and leads to results of the same completeness as in the exact solvable models. Second, the results which have been obtained earlier are in fact valid only for bosonic systems. The critical exponents in fermionic systems differ, generally speaking, from the bosonic ones and can also be obtained by means of the finite-size method. At last, the finite-size effects allow one to 
calculate the asymptotics of vacuum expectation values of some non-local operators in the $1 d$ systems. All these questions are the subject of the present paper.

We shall consider the following classes of $1 d$ systems. The first one contains the continuous models of the spinless Fermi- or Bose-gas with interaction of a general form, the second quantized Hamiltonian being (the mass of particle is $1 / 2$ ) :

$$
\hat{H}=\int_{0}^{L} d x \cdot \partial_{x} \psi^{*}(x) \partial_{x} \psi(x)+\frac{1}{2} \cdot g \iint_{0}^{L} d x d y \cdot \psi^{*}(x) \psi^{*}(y) V(x-y) \psi(x) \psi(y)
$$

Here $L$ is the length of the system, $V(x)$ is some even pairwise (repulsive) potential of a rather general form, $g>0$ is the coupling constant. The operators $\psi^{*}, \psi$ satisfy usual equal-time (anti)commutation relations (to indicate the statistics manifestly we sometimes use the notation $\psi_{B}$ or $\psi_{F}$ ). The number of particles in the system $N$ is conserved; in the thermodynamic limit $N \rightarrow \infty, L \rightarrow \infty$ and $\rho=N / L$ is the equilibrium density. Note that the potential $V(x)=\delta(x)$ admits the exact solution [12]. In the first quantized framework the Hamiltonian (7) has the form

$$
\hat{H}=-\sum_{i=1}^{N} \frac{\partial^{2}}{\partial x_{i}^{2}}+g \sum_{i<j}^{N} V\left(x_{i}-x_{j}\right) .
$$

Sometimes we use this language for convenience.

The second case under consideration is the lattice version of (7)-(8):

$$
\begin{aligned}
H= & -\sum_{x=1}^{L}\left[\psi^{\dagger}(x+1) \psi(x)+\psi^{\dagger}(x) \psi(x+1)-2 \psi^{\dagger}(x) \psi(x)\right] \\
& +g \sum_{x<y}^{L} \psi^{\dagger}(x) \psi(x) V(x-y) \psi^{\dagger}(y) \psi(y)
\end{aligned}
$$

Here we put the lattice spacing equal to 1 , so $x$ and $L$ become dimensionless quantities now. If necessary, one can easily restore dependence of the lattice spacing in final answers.

At last, we shall deal with $X X Z$ Heisenberg antiferromagnet (or simply the spin chain) with the lattice Hamiltonian:

$$
\hat{H}_{X X Z}=\frac{1}{2} \sum_{x=1}^{L}\left(\sigma_{x}^{1} \sigma_{x+1}^{1}+\sigma_{x}^{2} \sigma_{x+1}^{2}+\cos \gamma \sigma_{x}^{3} \sigma_{x+1}^{3}\right), 0 \leq \gamma<\pi
$$


Here $L$ is the number of sites of the lattice, $\sigma^{j}$ are usual Pauli matrices, $\gamma$ is the anisotropy parameter. Isotropic $(X X Z)$ Heisenberg antiferromagnet corresponds to $\gamma=0$. For the $X X Z$ antiferromagnet model there exists an exact solution. Namely, all eigenstates of the Hamiltonian (10) can be constructed explicitly by the Bethe's method [12]. The simplest eigenstate with all spins looking at the same direction (i.e. full spin is equal to $L / 2$ ) is the bare (unphysical) vacuum. The physical vacuum of the antiferromagnet has the minimal possible value of the full spin ( 0 or $1 / 2$ depending on the parity of number of sites $L$ ) and corresponds to the filled bare vacuum, the number of reversed spins being an analog of the number of particles in the models (7)-(9).

In fact, after the Jordan-Wigner transformation (that is, $\psi(x)=\left(\prod_{j=1}^{x-1} \sigma_{j}^{3}\right) \sigma_{x}^{-}, \psi^{\dagger}(x)=$ $\sigma_{x}^{+}\left(\prod_{j=1}^{x-1} \sigma_{j}^{3}\right)(10)$ can be considered as a special case of (9). The simplest Hamiltonian (10) is chosen to make possible a comparison with the Bethe ansatz results. We can also consider more general spin $1 / 2$ chains with the exchange interaction corresponding to arbitrary potentials in (9).

All these models have a number of common physical properties. The most important property is the absence of a gap in the low-energy spectrum. In fact, such a general statement is not quite correct because one correctly can tune $V(x)$ and $\rho$ to create a gap in the spectrum of the lattice system (9). We are convinced, however, that the generic case with spinless $1 d$ systems is just the gapless spectrum with unique sound velocity巴. Some comments on this point are contained in the Section 2.

Another important property of models under consideration is that the central charge calculated from (4) in $X X Z$ antiferromagnet is equal to $1[3,14]$ and just the same holds for the model (7)-(9). So all these models are described by the universality class of a Gaussian model [21]. It is not very amusing since the lowest excitations are nothing but free phonons.

Some comments on long-wave approximation are in order. The proper distance $x_{c}$ when individual particles have sense is equal to $x_{c} \sim L / N=1 / \rho$. At large distances $(x \gg$ $x_{c}$ ) there arises an effective theory describing the phonon excitations, the information

\footnotetext{
${ }^{1}$ As for the systems with internal degrees of freedom, they have in general a number of branches of gapless excitations with different sound velocities. This more complicated case is out of the scope of the present paper.
} 
about interaction at small distances being contained in the only parameter $v$. Then the low-energy excitations corresponding to the linear dispersion law lead to effective scaling and conformal invariance (the $O(2)$-invariance is trivially restored by the choice of units so that $v=1)$. Therefore, the deviations from the exact conformal theory can only be discovered at small $L$, so the series in $L^{-1}$ are correct.

Now we briefly describe the content of the paper. In the Section 2 we calculate the energies of low-lying excitations for the continuous Bose-gas model and obtain the spectrum of scaling dimensions for this system. The more complicated case of lattice systems is considered in the Section 3. Here the universal expressions for the "compactification radius" in the Gaussian model through thermodynamic parameters of the system will be obtained. Then, using all these results, we write down the asymptotical series for correlation functions (Section 4). The most important examples (pair density correlator and one-particle density matrix) are discussed in detail. It turns out that a simple generalization of the finite-size method allows one to find the asymptotics of vacuum expectation values of some non-local operators as it is demonstrated in Section 5. Here the main examples are some special non-local operators in the spin chain. At last, the Section 6 is devoted to the systems of Fermi-particles. We show how the finite-size method may be used to calculate the exact critical exponents in this case.

The Appendix is devoted to a non-trivial example of $1 d$ multi-particle systems with long-ranged interaction - the so-called Sutherland (or, Calogero) model [28]. First, we calculate the effective central charge from the low-temperature behaviour of the specific heat. Second, we compare the exact results for correlation functions in this model with the asymptotical series obtained from the CFT-approach. This model has never been discussed in the literature from the conformal point of view. It seems to us that the detailed exposition of this example could be useful.

\section{Scaling dimensions in the Bose-gas model}

Let us consider the Bose-gas described by the Hamiltonian (5). To obtain the scaling dimensions of different operators it is necessary to classify the low-lying excitations having zero energy at $L=\infty$. We imply periodic boundary conditions, i.e. the particles live on 
a circle of the length $L$.

The typical excitation spectrum of the $1 d$ spectrum is shown schematically in Fig.1. One can learn that it is gapless with the energy tending to zero when the momentum is equal to $2 \pi \rho m$ ( $m$ is integer). These properties hold for rather large class of both long-range and short-range potentials $V(x)$. Indeed, the cases of small and large constants $g$ in $(7)-(8)$ have been studied in the literature. In [24], where the case of small $g$ in Fermi systems was studied perturbatively, it was concluded that the correlators have a power-like behaviour and no gap emerges. The case of long-range $V(x)$ and large $g$ (strong repulsion) was considered in refs.[25,26,27]. In this case, the absence of the gap becomes quite obvious. Indeed, independently from $V(x)$, the particles form a regular "dynamical lattice" (the so-called Wigner crystal) with sound-type low-energy excitations. From the other hand, there exist several exactly solvable models which possess a gapless spectrum. These are, for example, the model with delta-shape potential and that with the potential $V(x)=x^{-2}$ (the Sutherland model [28]). Evidently, the gap will not appear when performing small deformations of the Sutherland potential which do not entail a qualitative rearrangement of the ground state.

We should investigate the spectrum when $L$ is large but finite. Then the spectrum is quasi-discrete with the energy gap $\sim L^{-1}$ (see Fig.2). We are interested in the states with zero energy at $L=\infty$. The states $|\phi\rangle$ corresponding to the primary conformal operators have the minimal energies in comparison with that of the neighbouring states. So each gapless branch of the spectrum "gives rise" to a primary operator. The higher excitations are the states of the conformal tower with $k>0$ or $\bar{k}>0$ in (1).

Certainly, we are able to calculate the energy $E_{L}^{\phi}$ of the states $|\phi\rangle$ when the physical states can be constructed explicitly. This is the case for the exactly solvable models [12], in particular, for the Bose-gas with $\delta$-shape interaction. But it turns out that in general case all that information in fact is not necessary and the energy $E_{L}^{\phi}$ of a "primary" state $|\phi\rangle$ may be obtained using simple thermodynamic reasoning.

Here we shall demonstrate this for the system (7)-(8). Let us begin with the excitations which conserve the number of particles. The simplest suitable excitation is the creation of a phonon. The minimal possible momentum is $\pm 2 \pi / L$ (the $\operatorname{dots} A_{0}, \bar{A}_{0}$ in Fig.2). Evidently, the energy is equal to $2 \pi v / L$, and the gap in the momentum spectrum is 
absent: $P_{\infty}^{\phi}=0$. Then $(1,2)$ imply $h= \pm s=1$. Let us denote $\phi_{ \pm}^{0}$ the corresponding primary operators. Then

$$
\left\langle\phi_{+}^{0}(x) \phi_{+}^{0}(0)\right\rangle=\left\langle\phi_{-}^{0}(x) \phi_{-}^{0}(0)\right\rangle \sim x^{-2} ; \quad\left\langle\phi_{+}^{0}(x) \phi_{-}^{0}(0)\right\rangle=\left\langle\phi_{-}^{0}(x) \phi_{+}^{0}(0)\right\rangle=0
$$

A more interesting excitation has the momentum $P_{\infty}^{\phi}=2 \pi \rho\left(A_{1}\right.$ in Fig.2). This excitation is produced by the "rotation" of the whole system with minimal angular momentum (i.e. it can be described by the first level of the "rotator"). In other words, this state can be produced from the ground state by transition to another inertial frame of reference. The periodic boundary conditions imply the quantization of the velocity of this new frame. More generally, one produces the family of the states $\left|\phi_{0, m}\right\rangle, m$ is an integer $\left(A_{2}\right.$ and so on in Fig.2). The momenta of these states are equal to $2 \pi \rho m: P_{0, m}=2 \pi \rho m$, and their energies can be obtained by considering the uniform motion of the whole system, the momentum of each particle being equal to $2 \pi m / L$ :

$$
\delta E_{0, m}=N(2 \pi m / L)^{2}=\frac{2 \pi v}{L} \cdot \frac{2 \pi \rho m^{2}}{v} .
$$

Comparing (3) and (12) we find the dimensions of the operators $\phi_{0, m}$ :

$$
h_{0, m}=2 \pi \rho v^{-1} m^{2}, m \in \mathbf{Z} ; s=0 .
$$

The above derivation may seem to be not quite correct since it neglects the quantum nature of the ground state. But more rigorous arguments lead to the same result. Indeed, let us turn to another inertial frame and use the first quantized language for convenience. Then the full ( $N$-particle) wave function is transformed as

$$
\Psi\left(\left\{x_{i}\right\}\right) \rightarrow \tilde{\Psi}\left(\left\{x_{i}\right\}\right)=\exp \left(i q \sum_{k=1}^{N} x_{k}\right) \cdot \Psi\left(\left\{x_{i}\right\}\right)
$$

This function should be single-valued, so the momentum $q$ is quantized: $q=2 \pi m / L$, $m \in \mathbf{Z}$. Now acting to $\tilde{\Psi}$ by the Schrödinger operator (8) one obtains (12).

The same result can be obtained also in the second quantization formalism. In this case one should work with the Hamiltonian (7) shifted by chemical potential term 


$$
\hat{H} \rightarrow \hat{H}-\mu \hat{N}
$$

where $\hat{N}=\int_{0}^{L} d x \psi^{\dagger}(x) \psi(x)$ is the operator of the number of particles,

$$
\mu=\left.\lim _{L \rightarrow \infty} \frac{\partial E_{L}^{v a c}}{\partial N}\right|_{\rho=c o n s t}
$$

is the chemical potential. The Galilean boost operator is

$$
\hat{K}=\int_{0}^{L} d x x \psi^{\dagger}(x) \psi(x)
$$

and the transition to another inertial frame can be described by

$$
\hat{H} \rightarrow e^{-i q \hat{K}} H e^{i q \hat{K}}
$$

We leave the calculation for the reader.

The states $\left|\phi_{ \pm}^{0}\right\rangle,\left|\phi_{0, m}\right\rangle$ are excitations in the sector with the fixed number of particles. Now let us consider the excitations which change the number of particles. Now one should work with the modified Hamiltonian (15). In Bose case the adding of $n$ particles results in the energy shift

$$
\delta E_{n, 0}^{B}=\frac{1}{2} n^{2}\left(\partial E_{L}^{v a c} / \partial N^{2}\right)=\frac{n^{2}}{2 L}\left(\partial^{2} \epsilon_{0} / \partial \rho^{2}\right)=\frac{2 \pi v}{L} \cdot \frac{v n^{2}}{8 \pi \rho},
$$

where $\epsilon_{0}$ again denotes the energy density. Here we used the well-known thermodynamic relation

$$
v^{2}=2 \rho\left(\partial^{2} \epsilon_{0} / \partial \rho^{2}\right)
$$

Now we can obtain the dimensions of the new family of primary operators $\phi_{n, 0}$ :

$$
h_{n, 0}=\frac{v n^{2}}{8 \pi \rho}, s=0 .
$$

Certainly, it is possible to combine the above excitations, i.e. to add $n$ particles and then to "rotate" the system at the $m-t h$ level. In this way we obtain the operators $\phi_{n, m}$ with the dimensions 


$$
h_{n, m}=\frac{n^{2}}{R^{2}}+\frac{m^{2} R^{2}}{4} ; n, m \in \mathbf{Z}
$$

where we denote

$$
R^{2}=8 \pi \rho v^{-1}
$$

The dimensions $h_{0,1}$ and $h_{1,0}$ have been obtained first in [3] (in this paper the case of $V(x)=\delta(x)$ was considered), where they were represented in terms of integrable systems theory. One can check that these dimensions coincide with ours, with the former being expressed through the sound velocity. It seems to us that the form of the answer given by (21)-(22) is more convenient, as it has sense for quite arbitrary potentials, and all the information about potential is contained in the unique parameter $v$ (or $R$ ). In the next section we shall obtain a universal expression for $\mathrm{R}$ valid for lattice systems as well.

In exactly solvable models one can calculate the central charge using the formula (2). Indeed, both the model with $V(x)=\delta(x)$ in (7)-(8) and $X X Z$-spin chain (10) provide $c=1$ [3]. In the Sutherland-Calogero model $\left(V(x)=x^{-2}-[28]\right)$ the dependence $f(T)$ at low temperature can be found using the results of [29], and (6) yields the answer $c=1$ too (see the Appendix). A more general argument is the following. Since the scaling dimensions depend on a continuous parameter, the central charge should be not less than 1 [30]. Then one can always extract an "irreducible theory" having $c=1$ from the initial theory [31]. On the other hand, the central charge greater than 1 implies the number of degrees of freedom greater than 1. However, it is impossible for the systems under consideration since we have assumed the gapless spectrum with the only branch of phonon excitations. The result $c=1$ seems to be quite natural as just such a theory describes the phonon system in the long-wave limit.

Note that the spectrum of scaling dimensions (21) also implies $c=1$. Indeed, roughly speaking, almost all conformal theories with $c=1$ are equivalent to the Gaussian models with the spectrum of dimensions like (21) which can be parameterized by the unique continuous parameter (the whole classification of those theories would include the Gaussian models with corresponding orbifold line and three isolated models in moduli space of the conformal theories [24]). 
More precisely, the Gaussian model is the $2 d$ free massless scalar field theory with the action (here $z=x+i y, \bar{z}=x-i y)$ :

$$
S_{R}=\frac{1}{2 \pi} \int d^{2} z \partial_{z} \varphi \partial_{\bar{z}} \varphi
$$

which is evidently $U(1)$-invariant. The field $\varphi(z, \bar{z})$ takes values in the "circle" of radius $R$, i.e. one identifies $\varphi$ and $\varphi+2 \pi R$. The spectrum of this theory is given by (21) $[21,32]$, with the parameter $R$ playing the role of the compactification radius in string theory. The operator $\phi_{+}^{0}\left(\phi_{-}^{0}\right)$ can be identified with the (anti)chiral $U(1)$-current $\partial_{z} \varphi\left(\partial_{\bar{z}} \varphi\right)$, and $\phi_{n, m}$ can be identified with the exponential of the free field:

$$
\phi_{n, m} \rightarrow: \exp \{i p \varphi+i \bar{p} \bar{\varphi}\}:
$$

where $: \ldots$ : denotes the proper normal ordering and the dimensions are

$$
\begin{gathered}
(\Delta, \bar{\Delta})=\left(p^{2} / 2, \bar{p}^{2} / 2\right), h=\Delta+\bar{\Delta}, s=\Delta-\bar{\Delta}, \\
(p, \bar{p})=\left(n R^{-1}+m R / 2, n R^{-1}-m R / 2\right) ; n, m \in \mathbf{Z} .
\end{gathered}
$$

This theory is invariant under the dual transformation $R \rightarrow 2 / R$, and $R=\sqrt{2}$ is the self-dual point corresponding to the isotropic $(X X X)$ antiferromagnet $(\gamma=0$ in $(10))$.

\section{Scaling dimensions in lattice models}

As we saw in the previous section, the continuous models have an exact symmetry which allows one to obtain the answer immediately, namely, they are invariant with respect to Galilean transformations. In the case of lattice models (9)-(10) the exact Galilean invariance is broken. Nevertheless, we shall see that it is possible to apply the finite-size method in this case too [43]. In this section the most general relations between critical exponents and thermodinamic parameters of the system will be obtained.

Consider the system of spinless particles on $1 d$ lattice with the Hamiltonian (9) modified according to (15). Then $\rho=\partial \epsilon_{0} / \partial \mu$ is the density of particles at zero temperature. We imply periodic boundary conditions in (9). It will be convenient to imagine the lattice 
as being rolled up into a ring. Again, we shall assume that there is no gap in the energy spectrum of the system. In this case our arguments do not depend on the specific form of $V(x)$.

The operators $\phi_{ \pm}^{0}$ can be constructed in the same way as in the continuous case and their dimensions and correlators are given by the same formulas (11).

Now we consider the analog of "rotating states" $\left|\phi_{0, m}\right\rangle$. By definition, $\left|\phi_{0, m}\right\rangle$ is the eigenstate of $H$ with momentum $2 \pi \rho m$ and minimal energy. Let us represent it in the form (it is convenient to work in the second quantization formalism in this case)

$$
\left.\left|\phi_{0, m}\right\rangle=\sum_{x_{j}} \exp \left(i p_{m}\right) \sum_{j+1}^{N} x_{j}\right) \Psi_{m}\left(x_{1}, \ldots, x_{N}\right) \prod_{k=1}^{N} \psi^{\dagger}\left(x_{k}\right)|0\rangle,
$$

where $p_{m}=2 \pi m L^{-1},|0\rangle$ is the bare vacuum, $N$ is the (conserved) number of particles. When $m=0\left|\phi_{0,0}\right\rangle=|v a c\rangle$ and $\Psi_{0}$ is the ground state coordinate wave function. We have extracted the exponential factor in $(27)$ in order to make $\Psi_{m}$ close to $\Psi_{0}$. This is really the case because $p_{m} \sim L^{-1}$ (note that in continuous systems $\Psi_{m}=\Psi_{0}$ for all $m$ ).

Acting to $\left|\phi_{0, m}\right\rangle$ by the Hamiltonian $H(9)$ one can see that $\Psi_{m}$ should be the ground state coordinate wave function (in the $N$-particle sector) of the following Hamiltonian $\hat{H}(p)$ at $p=p_{m}$ :

$$
\begin{aligned}
H(p)=H & +(1-\cos p) \sum_{x=1}^{L}\left(\psi^{\dagger}(x+1) \psi(x)+\psi^{\dagger}(x) \psi(x+1)\right. \\
& -i \sin p \sum_{x=1}^{L}\left(\psi^{\dagger}(x+1) \psi(x)-\psi^{\dagger}(x) \psi(x+1) .\right.
\end{aligned}
$$

Evidently, $H(p)$ is produced from $H=H(0)$ by the following transformation $\psi^{\dagger}(x) \rightarrow$ $\exp (i p x) \psi^{\dagger}(x), \psi(x) \rightarrow \exp (i p x) \psi(x)$. Due to the gauge invariance it means that the uniform magnetic field $B=p$ is applied to the system. In other words, $H(p)$ describes the system (9) in the uniform magnetic field orthogonal to our ring lattice (Fig.3). Note that the magnetic flux through the ring is equal to $2 \pi m$ (because $p$ is quantized: $p=p_{m}$ ).

Passing from $H$ to $H(p)$ we can write for the ground state energy shift $\epsilon_{m}$ (up to $L^{-1}$ ) the following general relation which is evident from the thermodynamic reasoning:

$$
\delta E_{0, m}=\epsilon_{m}=\frac{1}{2} L p_{m}^{2}\left(\frac{\partial^{2} \epsilon_{0}}{\partial p^{2}}\right)_{p=0}=2 \pi v L^{-1} \pi \eta v^{-1} m^{2}
$$


Here $\epsilon_{0}$ is the minimal eigenvalue of $H(p)$. When there is no Galilean invariance the current $j=i\left\langle\psi^{\dagger}(x+1) \psi(x)-\psi^{\dagger}(x) \psi(x+1)\right\rangle$ depends on momentum $p$ according to a non-linear relation. So we have introduced the "current-momentum" susceptibility $\eta$ in (29):

$$
\eta=\frac{\partial^{2} \epsilon_{0}}{\partial p^{2}}=\frac{\partial j}{\partial p}
$$

Of course, the same expression for the energy shift (29) may be obtained by means of the perturbation theory, with exact value of the $L^{-1}$ order term being given by the first two orders in $p$ (see $(28))$.

By comparing (3) and (29) we find the dimensions of the corresponding primary operators:

$$
h_{0, m}=\pi \eta v^{-1} m^{2}
$$

Another way to excite the system is to change the number of particles. Similarly to (18) we have in the Bose case

$$
\delta E_{n, 0}^{B}=\frac{1}{2} n^{2} L^{-1}\left(\frac{\partial^{2} \epsilon_{0}}{\partial \rho^{2}}\right)=\frac{1}{2} n^{2} \chi^{-1} L^{-1}
$$

where

$$
\chi=\frac{\partial^{2} \epsilon_{0}}{\partial \mu^{2}}=\frac{\partial \rho}{\partial \mu}
$$

is the usual susceptibility which is in a sense "dual" to $\eta$. (Note that in general (19) is no longer true!) Comparing with (3) we obtain a new family of primary operators with the following dimensions:

$$
h_{n, 0}=(4 \pi v \chi)^{-1} n^{2}
$$

Now let us note that the two susceptibilities $\eta, \chi$ are connected with the sound velocity $v$ by a universal thermodynamic relation. The simplest way to see this is to consider the wave equation for long waves. Repeating standard arguments leading to the wave equation and taking into account the relation between current and momentum one has 


$$
\chi \frac{\partial^{2} u(x, t)}{\partial t^{2}}=\eta \frac{\partial^{2} u(x, t)}{\partial x^{2}}
$$

(here $u(x, t)$ is the displacement of medium and $x$ should be considered as a continuous variable). Hence

$$
v^{2}=\eta \chi^{-1}
$$

Therefore, combining the above excitations and making use of (36) we find the spectrum of dimensions in the form (21), where

$$
R^{2}=4 \pi v \chi
$$

This is the general expression for the compactification radius and (due to (21)) for the critical exponents. Note that in the case of the exactly solvable Hubbard model the critical exponents were expressed through the susceptibility $\chi$ in the paper [44]. Let us consider some particular cases.

If the continuum limit is performed in (9) the exact Galilean invariance is restored. In this case

$$
\chi=\frac{2 \rho}{v^{2}}
$$

and (37) coincides with (22).

In the case of the $X X Z$ model (10) we have [14] (after re-interpretation of $\rho, \mu: \rho$ now is the total spin of the chain, $\mu$ is external magnetic field):

$$
\begin{gathered}
v=\frac{\pi \sin \gamma}{\gamma}, \\
\chi=\frac{\gamma}{2 \pi(\pi-\gamma) \sin \gamma} .
\end{gathered}
$$

Hence

$$
R^{2}=\frac{2 \pi}{\pi-\gamma}
$$

which coincides with the Bethe ansatz results. The finite size corrections and scaling dimensions were studied in the framework of Bethe ansatz in papers [14,45]. 


\section{Asymptotics of the correlation functions}

In this section we shall obtain the correlation functions of the models (7)-(10) in the asymptotic series form. The most important correlators are density-density correlator $H(x)=\langle\rho(x) \rho(0)\rangle, \rho(x)=\psi^{*}(x) \psi(x)$ being the density operator, and the one-particle density matrix $S(x)=\left\langle\psi^{*}(x) \psi(0)\right\rangle$. In the spin chain the similar objects are $H(x)=$ $\left\langle\sigma_{x}^{3} \sigma_{1}^{3}\right\rangle$ and $S(x)=\left\langle\sigma_{x}^{+} \sigma_{1}^{-}\right\rangle$, with $\sigma^{ \pm}=\sigma^{1} \pm i \sigma^{2}$.

The operators $\psi(x), \rho(x)$ have no definite conformal dimensions, since they do not behave properly under conformal transformations. Nevertheless, they are local operators and, as such, can be represented as linear combinations of the primary and descendant operators. For simplicity, we write this sum at the moment rather symbolically without accounting of the "descendant contributions" (their role will be discussed below). As we shall see, to obtain the leading asymptotics it is sufficient to take into account only the primary operator contributions. Then, the general expression for a local operator $\hat{O}(x)$ has a form

$$
\hat{O}(x)=\sum_{\phi} C_{\phi} \exp \left(i P_{\infty}^{\phi} x\right) \phi(x),
$$

where the sum runs over primary operators $\phi$ and $C_{\phi}$ are some numerical coefficients. A possible gap in the momentum spectrum leads to appearance of the oscillating factors $\exp \left(i P_{\infty}^{\phi} x\right)$ in this formula. To find the correlation functions of the type $\langle\hat{O}(x) \hat{O}(y)\rangle$ it is sufficient to calculate pair correlators of primary operators $\phi$ by using the well-known technique of conformal field theory $[10,33]$. The most convenient way to do this is to represent primary fields in the exponential form (24) and to average their products by performing the conventional Gaussian functional integrals with the action (23).

The coefficients $C_{\phi}$ are non-zero if $\langle v a c|\hat{O}(x)| \phi\rangle \neq 0$ in the thermodynamic limit (here $\langle v a c|$ is the physical vacuum; for brevity sometimes we merely use the brackets to denote the physical vacuum expectation value). For example, the results of the previous section yield the following series (for Bose statistics):

$$
\psi_{B}(x)=\sum_{m=-\infty}^{\infty} c_{m} \cdot \exp (2 \pi i \rho m x) \phi_{1, m}(x)
$$




$$
\begin{gathered}
\psi_{B}^{*}(x)=\sum_{m=-\infty}^{\infty} c_{m}^{*} \cdot \exp (-2 \pi i m \rho x) \phi_{-1,-m}(x), \\
\rho(x)=\phi_{+}^{0}(x)+\phi_{-}^{0}(x)+\sum_{m=-\infty}^{\infty} c_{m}^{\prime} \cdot \exp (2 \pi i m \rho x) \phi_{0, m}(x),
\end{gathered}
$$

In the last formula $\phi_{0,0}$ denotes the identity operator. Using these series one can directly obtain asymptotical expansions of the correlation functions $H(x)$ and $S(x)$ as well as similar multi-point correlators. Note that the terms $\left\langle\phi_{+}^{0}(x) \phi_{+}^{0}(0)\right\rangle=\left\langle\phi_{-}^{0}(x) \phi_{-}^{0}(0)\right\rangle \sim x^{-2}$ appear in the $\rho \rho$-correlator, with the corresponding exponent being equal to the canonical dimension of the operator $\rho$ (independently of the coupling constant). The asymptotical series for $H(x)$ always contains such term but in the most interesting examples this term is non-leading.

Now let us discuss the descendant contributions. Generally speaking, the expression (42) should be supplemented by the descendant operators for each primary one satisfying the above selection rule. The conformal Ward identities imply that the descendant operator $\hat{O}(x)$ in a correlation function can be substituted by a number of differentiations of the same correlator in which the primary field $\phi(x)$ stays instead of its descendant $\hat{O}(x)$. Thus, the descendant contributions correspond to the terms with exponents exceeding the leading ones by a positive integer. For example, the first descendant of $\phi_{0,1}$ gives a contribution to $H(x)$ of the form $x^{-\frac{1}{2} R^{2}-1} \sin (2 \pi \rho x)$. So, accounting for the descendants carefully, we are write down the whole asymptotical series $\left(x \gg \rho^{-1}\right)$ :

$$
\begin{gathered}
H(x)-\rho^{2}=\sum_{k=0}^{\infty} A_{k} x^{-2-k}+\sum_{m=1}^{\infty} x^{-\frac{1}{2} m^{2} R^{2}}\left[\sum_{k=0}^{\infty} A_{m, k} \cos \left(2 \pi m \rho x+\frac{\pi k}{2}\right) x^{-k}\right], \\
S(x)=\sum_{m=0}^{\infty} x^{-2 / R^{2}-\frac{1}{2} m^{2} R^{2}}\left[\sum_{k=0}^{\infty} B_{m, k} \cos \left(2 \pi m \rho x+\frac{\pi k}{2}\right) x^{-k}\right] .
\end{gathered}
$$

Here $A, B$ are some numerical coefficients.

The formulas (46),(47) are in a complete agreement with some exact results. These are the calculation of the density matrix in impenetrable boson system $(V(x)=\delta(x)$, $g=\infty$ in (7)-(8)) [34,35] and the exact result for the correlator $H(x)$ in the Sutherland model at the special value of the coupling constant $g=4$ [28]. These correlators do 
really have the form of $(46),(47)$ (Appendix). One can also compare these formulas with the answers of the papers [26,36], where the asymptotics of $H(x)$ and $S(x)$ was obtained by a direct calculation in case of the strongly repulsive particles with long-range interaction. In those papers the perturbation theory with respect to the small parameter $g^{-1}$, where $g$ is the coupling constant have been constructed. The result was that the parameter $R$ should be determined by the same expression (22) provided that all orders of the perturbation theory are taken into account. However, $R$ is small parameter when $g$ is large, so only the "primary" contributions (i.e. the terms with $k=0$ in $(46),(47)$ ) were essential in refs.[26,36]. The rest terms are negligible on the background of slowly decreasing contributions $\sim x^{-\frac{1}{2} m^{2} R^{2}}$. Just the same reason does not allow one to obtain the term $\sim x^{-2}$ in (46) by the method of ref.[26]. Meanwhile, there are models (e.g. the antiferromagnet with $\pi / 2<\gamma<\pi$ ) where this term becomes the leading one.

The last check of $(46),(47)$ is the calculation of non-equal-time correlators, both the charged and neutral ones, by a new exact method proposed in refs.[37]. To compare these results with ours one has to take into account spins of the operators and to consider the combinations $x \pm v t$ instead of $x$. If we suppose the symmetry with respect to exchange $x+v t \Leftarrow \Rightarrow x-v t$, we reproduce the answers of papers $[37,38]$.

Now we would like to discuss the meaning of the series $(46),(47)$ with unknown coefficients. At the first glance quite arbitrary function can be represented in such a form. Nevertheless, the expressions (46),(47) contain an important information.

First, it turns out that in all cases when the correlators can be obtained using other methods (see above) the coefficients $A, B$ quickly decrease when $k \rightarrow \infty$, so the series are well convergent. From the other hand, the above mentioned approaches as well as exact methods usually reproduce just several leading terms of (46),(47). Thus these expressions seem to be adequate to the problem. Second, the subseries corresponding to the descendants (the sums over $k$ in $(46),(47)$ ) can be rolled up to smooth analytic function, but the sum over primary fields contains the fractional degrees of $x$ :

$$
H(x)-\rho^{2}=\frac{f_{0}(x)}{x^{2}}+\sum_{m=1}^{\infty} f_{m}(x) \cdot x^{-m^{2} R^{2} / 2},
$$




$$
S(x)=\sum_{m=0}^{\infty} \xi_{m}(x) \cdot x^{-2 / R^{2}-m^{2} R^{2} / 2} .
$$

When $R^{2}$ is irrational, the number of different types of ramification is infinite $\left(x^{\alpha}\right.$ and $x^{\beta}$ give the same ramification if $\alpha$ and $\beta$ have the same fractional parts). It apparently implies the essential singularity. On the contrary, if $R^{2}$ is rational the correlators (48),(49) contain the finite number of the ramification types. This case corresponds to the rational conformal theories [39], and demonstrate the finiteness of the number of fields which are primary with respect to some large chiral algebra [40].

The question emerges whether the models described by the rational conformal theories in the long-wave limit have any peculiar properties. Our conjecture is that the ground state wave function in such models is analytic function of every variable on the finite covering of the complex plane (may be with puncture at $\infty$ ). Using the results of refs.[28,29] one can easily check that this is the case for the Sutherland modell?

Now we would like to discuss briefly some corrections to the expressions $(46),(47)$ originated from the deviations of the critical point Hamiltonian from the fixed point one. That is they can differ by the irrelevant operators which have their conformal dimensions $h_{\text {irrel }}$ greater than 2 (and, therefore, do not effect the critical behaviour). Then they induce contributions to $(46),(47)$ decreasing with the distance faster than the leading terms being suppressed by $x^{-h_{\text {irrel }}+2}$. Though the structure of series in this case is more complicated their global structure is the same in main features. In particular, the notion of rationality (the finiteness of the ramification types) remains unchanged. Certainly, in special cases the corrections merely ought not to appears as it is the case for impenetrable Bose-gas.

More interesting point is the possible logarithmic corrections to leading asymptotics. The reason of this is the appearance of the additional marginal operators (i.e. operators with dimension $(1,1)$ ) which might be given rise in critical Hamiltonian at some special values of coupling constants (and, therefore, $R$ ). As a rule, the logarithmic corrections emerge when the symmetry of the system increases [32]. For example, in the $S U(2)$ symmetrical case of isotropic antiferromagnet in zero magnetic field the leading term of

\footnotetext{
${ }^{2}$ We thank M.Olshanetsky and A.Perelomov for the discussion on this point.
} 
the correlator $\left\langle\sigma_{x} \sigma_{1}\right\rangle$ given by (46) should be multiplied by $(\ln x)^{\frac{1}{2}}[41][$. In less symmetrical models the multiplicative corrections to leading terms are absent, but they can appear in sub-leading terms.

To conclude this section two remarks are in order.

One can easily obtain the multi-point correlators using the expressions (43)-(45), as primary fields can be realized as exponentials of free scalar field and can be averaged directly with the Gaussian action $(c=1$ !). The final expression has a type

$$
\left\langle\prod_{i} \rho\left(x_{i}\right)\right\rangle \sim \sum_{\left\{m_{i}\right\}} c_{m_{i}} \exp \left(i 2 \pi \rho \sum m_{i}\right) \prod_{i<j}\left|x_{i}-x_{j}\right|^{-\frac{1}{2} m_{i} m_{j} R^{2}}+\text { non-leading terms }
$$

and should be compared with the one which can be obtained by the method of [36]. Then the primary field contributions are the same in both cases (descendant terms, as above mentioned, can not be taken into account by this method).

The second remark concerns the coefficients in the sums (43)-(47). Generally speaking, the arguments of trigonometric functions in (46),(47) should be added by some constant (or slowly varying) phase (if $c_{m} \neq c_{-m}, c_{m}^{*} \neq-c_{-m}^{*}, c_{m}^{\prime} \neq c_{-m}^{\prime}$ and so on). Nevertheless sometimes this phase is absent. For example, this is the case for (46) since $c_{m}^{\prime}=c_{-m}^{\prime}$ due to the evident symmetry with respect to the exchange $q \leftarrow \rightarrow-q$ in the wave function $\tilde{\Psi}$ of the other inertial frame. Here it would be appropriate to remark that the exponential operators $\phi_{0, m}+\phi_{0,-m}$ from (16) form closed operator algebra which corresponds to global $O(2)$-invariance instead of $U(1)$-invariance as it is in (23).

Thus, we have demonstrated that the long wave correlation properties of bosonic spinless systems can be described by the Gaussian model with proper "compactification radius" $R$. The parameter $R$ is defined by the general formula (37) which gives (22) and (41) in the cases of continuous bose-gas and Heisenberg antiferromagnet respectively.

\section{Vacuum expectation values of non-local operators}

In the main body of this section we shall consider the Heisenberg spin chain (10). Let us consider the following non-local operators in this model:

\footnotetext{
${ }^{3}$ We are indebted to V.E.Korepin who drew our attention to this paper.
} 


$$
S(x, y) \equiv \sum_{j=x}^{y} \sigma_{j}^{3}=\exp \{i \pi q(x, y)\},
$$

$q(x, y)$ being the operator of the number of reversed spins at the sites on the way from $x$ to $y$, and

$$
T(x, y)=P(x, x+1) P(x+1, x+2) \ldots P(y-1, y) P(y, x)
$$

where $P(x, y) \equiv 1 / 2\left(1+\sigma_{x} \sigma_{y}\right)$ is the operator of permutation of the spins at the sites $x$ and $y$. The operator $T(x, y)$ is simply the operator of cyclic permutation of the sites along the segment $[x, y]: x \rightarrow x+1, x+1 \rightarrow x+2, \ldots, y-1 \rightarrow y, y \rightarrow x$.

Our aim in this section will be to find the asymptotics of the following correlators $\langle v a c|S(x, y)| v a c\rangle,\langle v a c|T(x, y)| v a c\rangle,\langle v a c|S(x, y) T(x, y)| v a c\rangle$ at $|x-y| \gg 1$. Such nonlocal correlators are quite interesting objects from the point of view of the quantum inverse scattering method [42]. Another application of our results is the calculation of correlation functions in the system of fermions with spin 1/2 [27] where one should know the asymptotics of the above non-local correlators. Due to translational invariance these correlators depend only on $|x-y|$.

We shall show that these correlators have a power-law asymptotics. The corresponding exponents can be calculated using a natural generalization of the finite-size method. The idea is the following. As it was shown in [21], the (extended) Gaussian model has a non-local sector in which one should choose the numbers $n, m$ in (21) to be half-integers. The corresponding operators, e.g. $\phi_{0, \frac{1}{2}}$, are so-called disorder operators. They can be expressed through the free field $\varphi$ (see (23)) only in a non-local way. Acting at the ground state of the extended Gaussian model by such an operator, say $\phi_{0, \frac{1}{2}}$, one obtains a state in the sector with antiperiodic boundary conditions (b.c.). Therefore, the correlators of even number of disorder operators have sense in the sector with periodic b.c. A couple of disorder operators at the points $x, y$ in the spin chain looks like a non-local operator acting in the segment $[x, y]$. Certainly, that is what allows one to find the asymptotics of the vacuum expectation values of non-local operators by CFT.

Let us consider an extended Hilbert space containing all states of the spin chain with different number of sites and different b.c. simultaneously. We introduce the following 
operators acting in the extended space: the operator $a_{\beta}^{\dagger}(x)(\beta= \pm 1 / 2)$ creating a new site of spin $\beta$ between the sites $x$ and $x+1$ of the initial chain; the operator $b_{\beta}(x)$ annihilating the site $x$ (of $\operatorname{spin} \beta_{x}$ ) in the case of $\beta_{x}=\beta$ and giving zero if $\beta_{x}=-\beta$. Thus, $a_{\beta}^{\dagger}(x)$ acts from the sector of the extended Hilbert space corresponding to the chain with $L$ sites to the sector corresponding to $L+1$ sites. Quite similarly, $b_{\beta}(x)$ changes the number of sites from $L$ to $L-1$. The operator $b_{ \pm \frac{1}{2}}$ can be identified with non-local operator $\phi_{ \pm \frac{1}{2}, 0}$ in extended Gaussian model above. We also introduce the operator

$$
s(x) \equiv \prod_{j=x}^{L} \sigma_{j}^{3},
$$

which connects the sectors corresponding to periodic and antiperiodic b.c.

Indeed, let us consider the ground state wave function $\Psi\left(x_{1}, \ldots, x_{M}\right)$ of the antiferromagnet. Here $x_{k}$ are the coordinates (integer numbers) of reversed spins. For periodic b.c. one has $\Psi\left(x_{1}\right)=\Psi\left(x_{1}+L\right)$ with other $x_{k}$ being fixed (for brevity we write down explicitly only one variable). Let us denote $\tilde{\Psi}=s(x) \Psi$. In order to find $\tilde{\Psi}\left(x_{1}+L\right)$ we shall move $x_{1}$ and keep other variables fixed. Suppose we have $k$ reversed spins to the right from the site $x$. Then one can write for $x_{1}<x$ :

$$
\tilde{\Psi}\left(x_{1}\right)=s(x) \tilde{\Psi}\left(x_{1}\right)=(-1)^{k} \Psi\left(x_{1}\right)
$$

For $x_{1}>x$ one has the following evident equalities:

$\tilde{\Psi}\left(x_{1}+L\right)=s(x) \Psi\left(x_{1}+L\right)=(-1)^{k+1} \Psi\left(x_{1}+L\right)=(-1)^{k+1} \Psi\left(x_{1}\right)=-s(x) \Psi\left(x_{1}\right)=-\tilde{\Psi}\left(x_{1}\right)$.

So $\tilde{\Psi}$ belongs to the sector with antiperiodic b.c.

Now we can write

$$
\begin{gathered}
T(x, y)=\sum_{\beta} a_{\beta}^{\dagger}(x) b_{\beta}(x), \\
S(x, y)=s(x) s(y) .
\end{gathered}
$$

Let us denote by $|L,+\rangle(|L,-\rangle)$ the ground state of the spin chain with $L$ sites and periodic (antiperiodic) b.c. (in the thermodynamic limit these states coincide with $|v a c\rangle$, but we 
are interested in finite size corrections $\sim L^{-1}$ ). Working with extended Hilbert space we can consider the ground states in the sectors with another $L$ and antiperiodic b.c. as excited states over $|v a c\rangle \equiv|L,+\rangle$. Roughly speaking, $a_{\beta}^{\dagger}(x)$ creates an excitation with spin $1 / 2$ ("one half of magnon"). If $L$ is even then only even number of such excitations can exist, and if $L$ is odd then there necessarily exist odd number of such "half-magnons". Clearly. in the latter case the ground state is two-fold degenerate.

One can easily check that

$$
\left\langle L-1,+\left|b_{s}(x)\right| L,+1\right\rangle \neq 0,\left\langle L+1,+\left|a_{s}^{\dagger}(x)\right| L,+\right\rangle \neq 0,\langle L,-|s(x)| L,+\rangle \neq 0,
$$

in the thermodynamic limit, i.e. the states $|L \pm 1, \pm\rangle$ satisfy to the selection rule of Section 3. This means that in order to find the asymptotics at $|x-y| \gg 1$ of $\langle v a c|T(x, y)| v a c\rangle=2\left\langle a_{+\frac{1}{2}}^{\dagger}(x) b_{+\frac{1}{2}}(y)\right\rangle$ (obviously, $\left\langle a_{+\frac{1}{2}}^{\dagger} b_{+\frac{1}{2}}(y)\right\rangle=\left\langle a_{-\frac{1}{2}}^{\dagger}(x) b_{-\frac{1}{2}}(y)\right\rangle$ ) and $\langle v a c|S(x, y)| v a c\rangle=\langle v a c|s(x) s(y)| v a c\rangle$ we can use the formula (4) with the scaling dimensions being determined from the relation (3). It is sufficient to calculate the energy shifts of the states $|L \pm 1,+\rangle$ and $|L,-\rangle$ up to the first order in $L^{-1}$ and find their momenta. This can be done using the well-known exact solution (Bethe-ansatz) [12,14]. Certainly, one should modify the Hamiltonian as follows: $\hat{H}_{X X Z} \rightarrow \hat{H}_{X X Z}-\epsilon_{0} L$ where $\epsilon_{0}$ is the energy per one site. We omit these lengthy calculations here.

The results are as follows. The spectrum of scaling dimensions is given by the formula (21) with half-integer $n, m$. The parameter $R$ is determined according to (41). The correlation functions look like

$$
\begin{gathered}
<v a c|S(x, y)| v a c>=<v a c|\exp \{i \pi q(x, y)\}| v a c>\sim \cos [\pi(x-y) / 2] \cdot|x-y|^{-\lambda}, \\
<\operatorname{vac}|T(x, y)| v a c>\sim|x-y|^{-\mu}, \\
<v a c|T(x, y) \exp \{i \pi q(x, y)\}| v a c>\sim \cos [\pi(x-y) / 2] \cdot|x-y|^{-\lambda-\mu} .
\end{gathered}
$$

where 


$$
\mu=R^{-2} / 2, \lambda=R^{2} / 8
$$

Note that in the case of isotropic $(X X X)$ spin chain $(\gamma=0)$ the operator $s(x)$ has scaling dimension $1 / 8$ and coincides with the spin field [32].

The correlators (59)-(61) appear in the calculation of correlation functions in the multiparticle spin 1/2 systems as auxiliary objects. In particular, the result (61) was used in [46] for finding the long-wave asymptotics of the density matrix in the system of spin 1/2 Fermi particles with strong interaction.

To conclude this section let us make a remark on the corresponding non-local operators in the Bose-gas model (7)-(8). An analog of $S(x, y)$ is defined by the same formula

$$
S(x, y)=\exp [i \pi q(x, y)]
$$

where $q(x, y)$ is now the operator of number of particles in the segment $[x, y]$. The leading

term of $\langle S(x, y)\rangle\left(|x-y| \gg \rho^{-1}\right)$ can be obtained using the above technique:

$$
<\operatorname{vac}|S(x, y)| v a c>\sim \cos (\pi \rho|x-y|) \cdot|x-y|^{-R^{2} / 8}
$$

It is convenient to introduce an analog of the operator $s(x)(53)$ :

$$
s(x)=\exp [i \pi q(x, L)]
$$

which will be useful in the next section. The meaning of the operator $T(x, y)$ in the continuous case is obscure.

\section{Critical exponents in fermionic systems}

So far, dealing with the model (7)-(8), we implied the case of Bose-statistics. It is known that the critical exponent of the fermionic field correlator $S_{F}(x)=\left\langle\psi_{F}^{*}(x) \psi_{F}(0)\right\rangle$ differs from that of the bosonic one $S_{B}(x)=\left\langle\psi_{B}^{*}(x) \psi_{B}(0)\right\rangle[26]$. In this section we shall obtain the answer in the framework of the finite-size method.

There are two different approaches to this problem. The first one makes use of the fact that the operator $s(x)(65)$ produces the Jordan-Wigner transformation from bosonic 
field operators to the fermionic ones:

$$
\begin{aligned}
& \psi_{F}(x)=\psi_{B}(x) s(x), \\
& \psi_{F}^{*}(x)=s^{*}(x) \psi_{B}^{*}(x) .
\end{aligned}
$$

Following the line of the previous section we obtain the result:

$$
S_{F}(x) \sim \cos (\pi \rho x) \cdot x^{-2 / R^{2}-R^{2} / 8}
$$

It is also easy to write down the whole asymptotical series for $S_{F}(x)$ and see that it is in a good agreement with that of the paper [36] obtained by completely different methods.

Less formal arguments are the following. Let $\psi\left(x_{1}, \ldots, x_{N}\right)$ be the ground state wave function of the system (7) or (8) (in the first quantization language). It should be an eigenfunction of the full momentum $\hat{P}$ with an eigenvalue $P$. So we can write

$$
\exp (i \hat{P} a) \cdot \Psi\left(x_{1}, \ldots, x_{N}\right)=\Psi\left(x_{1}+a, \ldots, x_{N}+a\right)=\exp (i P a) \cdot \Psi\left(x_{1}, \ldots, x_{N}\right),
$$

where $a=L / N$. On the other hand, the cyclic permutation $x_{i} \rightarrow x_{i+1}$ results in the sign factor:

$$
\Psi\left(x_{2}, x_{3}, \ldots, x_{N}, x_{1}\right)=(-1)^{N-1} \Psi\left(x_{1}, \ldots, x_{N}\right)
$$

Setting $x_{1}=x, x_{2}=x+a, x_{3}=x+2 a, \ldots, x_{N}=x+(N-1) a$ and comparing these formulas we obtain the following selection rule for the full momentum:

$$
\exp (i P L / N)=(-1)^{N-1}
$$

or, writing $P=2 \pi \rho m$, as before,

$$
(-1)^{2 m}=(-1)^{N-1}
$$

\footnotetext{
${ }^{4}$ Here we use the fact that the ground state wave function $\Psi(x, x+a, x+2 a, \ldots)$ is non-zero everywhere (as it have no nodes at all).
} 
Thus, when $N$ is even, the ground state is two-fold degenerate $(P= \pm \pi \rho)$. In the case of free fermions this fact is obvious.

Clearly, the neutral excitations with zero energy ("rotations" of the system; see Sect.2) should have momenta $P_{0}+2 \pi m \rho(m \in \mathbf{Z})$ where $P_{0}$ is the ground state momentum. Thus, the dimensions of $\phi_{0, m}$ are given by the same formula (13). It is easy to see that this is also the case when we add even number of particles to the ground state. To avoid misunderstanding it should be stressed that the dimensions in bosonic and fermionic cases are generally not equal to each other, the point is that the sound velocity may be different in the two cases.

But the addition of an old number of particles $n$ changes the parity of $N$, so, according to $(71)$, we have for $n=1$ :

$$
\delta E_{1,0}^{F}=2 \pi v L^{-1}\left(R^{-2}+R^{2} / 16\right)=2 \pi v L^{-1} h_{1, \frac{1}{2}},
$$

where we have formally used the notation from (21).

Combining the different excitations in accordance with (71), we obtain the general form of scaling dimensions in the fermionic systems. The spectrum is given by the formula (21) with the following conditions on $n$ and $m$ :

a) when $n \in 2 \mathbf{Z}, m \in \mathbf{Z}$;

b) when $n \in 2 \mathbf{Z}+1, m \in \mathbf{Z}+1 / 2$,

$$
\text { i.e. } \quad m=m^{\prime}+\frac{1}{2}, m^{\prime} \text { is integer. }
$$

\section{Concluding remarks}

In this paper we have demonstrated how one can find the long-wave asymptotics of various correlation functions in one-dimensional field-theoretical models using the finite-size technique in CFT. The obtained results are quite general. They are valid both for integrable and non-integrable models with interaction of a general form.

In conclusion, we would like to say a few words about the meaning of our results. We have shown that the long-wave correlation properties of the wide class of one-dimensional 
spinless systems are described by the Gaussian model, the simplest conformal field theory. The Gaussian model still contains a free parameter — "compactification radius" $R$.

An important point is that the value of $R$ is determined by the nature of the short-range interaction (of the order of the mean distance between the particles $x_{c}$ ). So the shortrange properties of the system are included into the effective long-range CFT through the only one parameter.

One of the main results of this paper is the expression for $R$ in concrete systems through their thermodynamic quantities. Clearly, it immediately gives us all the critical exponents as functions of thermodynamic parameters of the system. At last, for a given system it is much easier to solve the thermodynamics than to calculate correlation functions directly.

We are grateful to V.E.Korepin, A.Yu.Morozov and A.A.Ovchinnikov for fruitful discussions.

\section{Appendix}

Let us consider as an example the system of the type (8) with

$$
V(x)=x^{-2}
$$

or

$$
V(x)=\frac{(2 \pi / L)^{2}}{\sin ^{2}(2 \pi x / L)}
$$

(in the thermodynamic limit (A1) is equivalent to (A2)). The model (A1) is called the Calogero model, (A2) — the Sutherland model [28]. We call its thermodynamic limit the Sutherland-Calogero (SC) model.

In order to find the effective central charge we should investigate the behaviour of the free energy $f(T)$ at low temperature $T \rightarrow 0$. The thermodynamics of SC model was studied in [29].

First, we have the general thermodynamic relation

$$
f(T)=\mu \rho-P
$$


where $P$ is the pressure. In [29] it was shown that the thermodynamics of SC-model is described by the system of equations

$$
\begin{gathered}
\pi \beta^{\frac{1}{2}} \rho=\int_{x_{0}}^{\infty} \phi^{\frac{1}{2}}(x) e^{x} \frac{d x}{\left(e^{x}+\alpha\right)^{2}}, \\
3 \pi \beta^{\frac{3}{2}} P=2 \int_{x_{0}}^{\infty} \phi^{\frac{3}{2}}(x) e^{x} \frac{d x}{\left(e^{x}+\alpha\right)^{2}},
\end{gathered}
$$

where $\beta=T^{-1}, \alpha=(1+\sqrt{1+2 g}) / 2(g$ is the coupling constant in (8)),

$$
\phi(x) \equiv \beta \mu+x+(1-\alpha) \ln \left(1+e^{-x}\right)
$$

For finding power-like corrections in $\beta^{-1}$ at $\beta \rightarrow \infty$ we can put $x_{0}=-\infty$ and expand sqrt $\phi$ in a power series. Let

$$
I_{n} \equiv I_{n}(\alpha)=\int_{-\infty}^{\infty} d x \frac{e^{x}}{\left(e^{x}+\alpha\right)^{2}}\left[x+(1-\alpha) \ln \left(1+e^{-x}\right)\right]^{n},
$$

then we rewrite (A4) up to $\beta^{-2}$ as follows:

$$
\begin{gathered}
\alpha \pi \rho=m u^{\frac{1}{2}}+\frac{\alpha I_{1}}{2 \beta \mu^{\frac{1}{2}}}-\frac{\alpha I_{2}}{2 \beta^{2} \mu^{\frac{3}{2}}}, \\
\frac{3}{2} \alpha \pi \rho=m u^{\frac{3}{2}}+\frac{3 \alpha \mu^{\frac{1}{2}} I_{1}}{2 \beta}+\frac{3 \alpha I_{2}}{8 \beta^{2} \mu^{\frac{1}{2}}} .
\end{gathered}
$$

The computation of the integrals gives

$$
I_{1}=0, I_{2}=\frac{\pi^{2}}{3}
$$

then

$$
f(T)=\frac{\alpha^{2} \pi^{2} \rho^{3}}{3}-\frac{T^{2}}{12 \alpha \rho}+o\left(T^{2}\right) .
$$

The sound velocity is $[28,29]$

$$
v=2 \pi \alpha \rho
$$

so, comparing with (6), we conclude that $c=1$. 
The exact density-density correlation function $H(x)$ is known in this model at $g=4$ [28]:

$$
H(x)=F^{2}(2 x)-\frac{1}{2} F^{\prime}(2 x) \int_{0}^{2 x} d z F(z)+1
$$

where

$$
F(x)=\frac{\sin \pi x}{x}
$$

(we put $\rho=1$ ). In this case $\alpha=2$ and $R^{2}=2$.

Expanding (A13) in a power series of $x^{-k}$ we obtain exactly the series (46) which follows from the CFT.

\section{References}

1. Blöte H.W., Cardy J.L., Nightingale M.P. Phys.Rev.Lett. 56 (1986) 742

2. Affleck I. Phys.Rev.Lett. 56 (1986) 746

3. Bogolubov N.M., Isergin A.G., Reshetikhin N.Yu. Pis'ma v ZhETF, 44 (1986) 405;

J.Phys.Math.Gen. A20 (1987) 5361

4. de Vega H.J., Karowski M. Nucl.Phys. B285 [FS19] (1987) 619

5. von Gehlen G., Rittenberg V. J.Phys. A20 (1987) 2577

6. Alcaraz F., Barber M., Batchelor M. Ann.Phys. 182 (1988) 280

7. Efetov K.B., Larkin A.I. ZhETF, 69 (1975) 764

8. Luther A., Peshel I. Phys.Rev. B12 (1975) 3908

9. Haldane F.D.M. J.Phys. C14 (1981) 2585

10. Belavin A.A., Polyakov A.M., Zamolodchikov A.B. Nucl.Phys. B241 (1984) 333

11. Cardy J.L. Nucl.Phys. B270 [FS16] (1986) 186

12. See, for example, Thacker H.B. Rev.Mod.Phys. 53 (1981) 253

13. Bogoliubov N.M., Izergin A.G., Reshetikhin N.Yu. J.Phys. A20 (1987) 5361

14. Alcaraz F., Barber M., Batchelor M. Ann.Phys. 182 (1988) 280

15. Alcaraz F., Baake M., Grimm U., Rittenberg V. J.Phys. A21 (1988) L117

16. Woynarovich F. Phys.Rev.Lett. 59 (1987) 259 
17. Berkovich A., Murthy G. Stony Brook preprint, ITP-SB-88-5, 1988

18. Alcaraz F., Martins M. J.Phys. A21 (1988) 1

19. Johannesson H. J.Phys. A21 (1988) L611

20. Izergin A.G., Korepin V.E., Reshetikhin N.Yu. Stony Brook preprint ITP-SB-88-47, 1988

21. Kadanoff L.P. Ann.Phys. 120 (1979) 39

Kadanoff L.P., Brown A.C. Ann.Phys. 121 (1979) 318

22. Mironov A.D., Zabrodin A.V. J.Phys. A23 (1990) L493

23. Mironov A.D., Zabrodin A.V. Phys.Rev.Lett. 66 (1991) 534

24. Krivnov V.Ya., Ovchinnikov A.A. JETP, 49 (1979) 328

25. Krivnov V.Ya., Ovchinnikov A.A. JETP, 55 (1982) 1628

26. Ovchinnikov A.A., Zabrodin A.V. JETP, 63 (1986) 1326

27. Ovchinnikov A.A., Zabrodin A.V. Phys.Lett. A130 (1989) 139

28. Sutherland B. J.Math.Phys. 12 (1971) 246,251

Calogero F. J.Math.Phys. 10 (1969) 2191

29. Krivnov V.Ya., Ovchinnikov A.A. Teor.Math.Phys. 50 (1982) 155

30. Friedan D., Qiu Z., Shenker S. Phys.Rev.Lett. 52 (1984) 1575

31. Cardy J.L. J.Phys. A20 (1987) L891

32. Dijkgraaf R., Verlinde E., Verlinde H. Comm.Math.Phys. 115 (1988) 649

33. Dotsenko Vl.S., Fateev V.A. Nucl.Phys. B240 [FS12] (1984) 312

34. Vaidya H.G., Tracy C.A. Phys.Rev.Lett. 42 (1979) 3

35. Jimbo M., Miwa T., Mori Y., Sato M. Physica, D1 (1980) 80

36. Zabrodin A.V., Ovchinnikov A.A. ZhETF, 88 (1985) 1233

37. Its A.R., Isergin A.G., Korepin V.E., Slavnov N.A. Australian National University preprint, IC/89/107, 120, 139

38. Bogoliubov N.M., Izergin A.G., Korepin V.E. Nucl.Phys. B275 [FS17] (1986) 687

39. Moore G., Anderson G. Rationality in conformal field theory. IASSNS-HEP-87/69

40. Moore G., Seiberg N. Nucl.Phys. B313 (1989) 16

41. I.Affleck, D.Gepner, H.J.Schulz, T.Ziman J.Phys. A22 (1989) 511

42. Korepin V.E. Funk.Analiz i priloz. 23 (1989) 15

43. Zabrodin A.V. JETP Lett., 51 (1990) 614 
44. Bogoliubov N.M., Korepin V.E. Int.Journ.Mod.Phys. B3 (1989) 427

45. Pokrovsky S.L., Tsvelik A.M., ZheTF, 93 (1987) 2232

46. Zabrodin A.V., Ovchinnikov A.A. Teor.Mat.Fiz. 85 (1990) 443 


\section{Figure Captions}

Fig.1 The excitation spectrum of $1 d$ spinless Bose-gas (schematically)

Fig.2 The spectrum of the same system in a finite volume

Fig.3 The lattice system in the uniform magnetic field 